\title{
The Changing Pattern of Published Articles in the Field of Otology: 1818 to Today
}

\author{
Jang Hee Park ${ }^{1}$, Janet Ren Chao ${ }^{2}$, Min Jae Kim ${ }^{1}$, Yoo Yeon $\mathrm{Kim}^{3}$, and Jun Ho Lee ${ }^{1,4}$ \\ ${ }^{1}$ Departments of Otorhinolaryngology-Head and Neck Surgery, ${ }^{3}$ Medical Genetics, College of Medicine, Hallym University, \\ Chuncheon, Korea \\ ${ }^{2}$ School of Medicine, The George Washington University, Washington D.C., USA \\ ${ }^{4}$ Institute of New Frontier Research, College of Medicine, Hallym University, Chuncheon, Korea
}

Received June 22,2017

Revised July 14, 2017

Accepted August 8, 2017

\author{
Address for correspondence \\ Jun Ho Lee, MD, PhD \\ Department of Otorhinolaryngology- \\ Head and Neck Surgery, \\ Chuncheon Sacred Heart Hospital, \\ College of Medicine, \\ Hallym University, \\ 77 Sakju-ro, Chuncheon 24253, \\ Korea \\ Tel $+82-33-240-5181$ \\ Fax +82-33-241-2909 \\ E-mail zoonox@nate.com
}

To examine and identify the changing pattern of published articles in the otology literature over the past several decades. We used a variety of search engines available through PubMed.gov based on key words in the following categories: diagnosis, diagnostic tools, and therapeutic tools. The number of searchable key words increased from 1945 to the present. Overall, there has been a major shift in topics cover in the otological literature. Most recently, there has been significant increases in the following categories: age-related hearing loss, speech discrimination, and cochlear implantation. Based on this analysis, we believe these represent the areas of major research in the field of otology today. The present study is a bibliometric analysis of the changing pattern of published articles using a new analytic approach. The results identify the shifting topics of research in otology and might be helpful for future studies in Otorhinolaryngology Head and Neck Surgery.

J Audiol Otol 2017;21(3):160-165

\section{Introduction}

Using the keyword "ear," one hundred sixteen thousand articles can be viewed on PubMed. The first article the keyword "ear" keyword in the literature is "Observations on some points relating to the Physiology and Pathology of the Ear" [1]. This article was published in 1818 in the journal: "Medico-Chirurgical Transactions." Following publication, only a few articles relevant to Otolaryngology were published until 1944. Suddenly, following the conclusion of World War II, in 1945 , the numbers of published articles were increased explosively. Since then, the total number of published studies has continued to increase continually and the areas of focus have shifted dramatically. Whether researchers are university-, community-, or institution-based, understanding the areas of grow-

This is an Open Access article distributed under the terms of the Creative Commons Attribution Non-Commercial License (http://creativecommons.org/licenses/by-nc/4.0/) which permits unrestricted non-commercial use, distribution, and reproduction in any medium, provided the original work is properly cited. ing interest and the questions being raised through published articles will help guide their future studies.

To address the need for researchers to recognize current trends, we quantified the evolving pattern of published articles by decade. To accomplish this, we used a bibliometric approach which has been employed by a few other investigators in the otolaryngologic field, but modified the research methodology [2-7]. Specifically, we employed specific word searches as a means to quantify the frequency with which an idea was examined, and to provide insight into the exact pattern of change over time. This methodology will help researchers, particularly those early in their careers, wanting to identify the various research opportunities that exist.

\section{Subjects and Methods}

\section{Data sampling}

We analyzed data from the PubMed.gov (National Center for Biotechnology Information, U.S. National Library of Med- 
icine, 8600 Rockville Pike, Bethesda MD, 20894 USA) website. We used the available search engine and entered a variety of the most common diseases and problems most relevant to otolaryngology including: vestibular schwannoma, vestibular neuritis, labyrinthitis, ototoxic hearing loss, temporal bone fracture, sudden hearing loss, otosclerosis, chronic otitis media, hereditary hearing loss, tinnitus, noise induced hearing loss, age related hearing loss, cholesteatoma, acute otitis media, benign paroxysmal positional vertigo (BPPV), Meniere's disease, otitis media, mastoiditis, and Eustachian tube dysfunction. Such diseases entities were based on otorhinolaryngology textbook (ISBN: 893370-5589, Otorhinolaryngology Head and Neck Surgery, ilzogak, 2009, Korea).

The totality of published articles per year was downloaded from 'Results by year.' The frequency of mention for each disease was entered into an Excel spreadsheet, and the patterns were analyzed and stastically correlated. Other, less common diseases in otology were searches as well, but the numbers of "results by year" were relatively small, so they were excluded from the present study.

In addition to using the various diseases as search words, various diagnostic tools in otology and otologic treatment mo- dalities were also examined and quantified.

\section{Pattern analysis of published articles over time}

From 1818 to July 2016, the number of articles published in each category was quantified. Given the huge number in each subheading, it would have been impossible to compare them for duplication. For this reason, percentages were used to compare the relative frequency over time. We assumed that the amount of overlap was comparable both within and across diseases, diagnostic tools, and treatments.

The data from each of the categories was then combined into a single percentage linear graph and represented geometrically based on area. The increasing or decreasing pattern for each search word was then compared and evaluated.

\section{Statistical analysis}

All of the data, were tested by spearman correlation coefficient test to get rho value (Table 1). Statistical analyses were carried out using SPSS version 21 (IBM Corp., Armonk, NY, USA) for Windows. $p<0.05$ was considered to be statistically significant for the analysis.

Table 1. The $p$ and rho values of each searching word using the spearman correlation coefficient analysis

\begin{tabular}{|c|c|c|c|c|c|c|c|c|}
\hline \multicolumn{3}{|c|}{ Diseases } & \multicolumn{3}{|c|}{ Diagnostic tools } & \multicolumn{3}{|c|}{ Treatments } \\
\hline & $p$ & Rho & & $p$ & Rho & & $p$ & Rho \\
\hline V schwannoma & $<0.001$ & 0.632 & Tuning fork test & 0.014 & 0.290 & Mastoidectomy & 0.303 & -0.123 \\
\hline$\checkmark$ neuritis & $<0.001$ & 0.537 & PTA & 0.114 & 0.189 & Stapes surgery & 0.037 & -0.246 \\
\hline Labyrinthitis & 0.009 & -0.304 & SD & $<0.001$ & 0.409 & Ossiculoplasty & $<0.001$ & 0.836 \\
\hline Ototoxic HL & $<0.001$ & 0.911 & IA & 0.001 & 0.397 & Tympanoplasty & 0.073 & -0.212 \\
\hline Temporal bone FX & 0.490 & 0.083 & EcoG & 0.001 & 0.381 & Myringoplasty & 0.822 & 0.027 \\
\hline Sudden HL & $<0.001$ & 0.826 & $A B R$ & $<0.001$ & 0.692 & $\checkmark$ tube & $<0.001$ & 0.882 \\
\hline Otosclerosis & $<0.001$ & -0.943 & ASSR & $<0.001$ & 0.878 & BAHA & $<0.001$ & 0.914 \\
\hline COM & 0.006 & -0.320 & OAE & $<0.001$ & 0.856 & $\mathrm{Cl}$ & $<0.001$ & 0.946 \\
\hline Hereditary HL & $<0.001$ & 0.486 & Newborn screening & $<0.001$ & 0.555 & Hearing Aid & 0.023 & 0.660 \\
\hline Tinnitus & $<0.001$ & 0.852 & ENG & $<0.001$ & -0.577 & & & \\
\hline Noise HL & 0.047 & 0.235 & Calori test & $<0.001$ & -0.486 & & & \\
\hline Aged HL & $<0.001$ & 0.972 & VOR & 0.761 & -0.037 & & & \\
\hline Chole & $<0.001$ & -0.589 & Posturography & $<0.001$ & 0.925 & & & \\
\hline$A O M$ & 0.057 & 0.226 & VEMP & $<0.001$ & 0.828 & & & \\
\hline BPPV & $<0.001$ & 0.949 & & & & & & \\
\hline Meniere & $<0.001$ & -0.890 & & & & & & \\
\hline OME & $<0.001$ & 0.623 & & & & & & \\
\hline Mastoiditis & $<0.001$ & -0.885 & & & & & & \\
\hline E tube dysfunction & $<0.001$ & 0.420 & & & & & & \\
\hline
\end{tabular}

Ototoxic HL, Otosclerosis, Noise HL, E tube dysfunction, EcoG, ABR, OAE, Newborn screening, ENG, Postulography, VEMP, Stapes surgery, Tympano, Myringo, $V$ tube, and BAHA were normal distribution. $p$ and rho means values of spearman correlation coefficient analysis. V: vestibular, HL: hearing loss, FX: fracture, COM: chronic otitis media, Chole: cholesteatoma, AOM: acute otitis media, BPPV: benign paroxysmal positional vertigo, OME: otitis media with effusion, E: Eustachian, PTA: pure tone audiometry, SD: speech discrimination, IA: impedence audiometry, ECOG: electrocochleography, ABR: auditory brainstem response, ASSR: auditory steady state response, OAE: otoacoustic emission, ENG: electronystagmography, VOR: vestibule-ocular response, VEMP: vestibular evoked myogenic potential, $\vee$ tube: ventilation tube, BAHA: bone anchored hearing aid, Cl: cochlear implant 


\section{Results}

\section{Published articles per year during past decade}

In general, the number of articles associated with each search word increased over time from 1945 to the present. Before 1945, the number of published articles was minimal, and therefore excluded from the analysis (Fig. 1A). The search word associated with the greatest number of articles was 'speech discrimination.' And the most frequently published subdivisions were "age related hearing loss" for diagnosis, "speech discrimination" for diagnostic tool, "hearing aid" and "cochlear implantation" for management and treatment.

To identify the relative growth of published otologic articles in all journals over time, the relative number in each category was graphed over time and combined into single figure.
The changing pattern of research by disease category

Fig. 1B demonstrates the changing pattern of published articles by disease category. From 1946 to 1960's, mastoiditis, Meniere's disease and otosclerosis were the main topics found in the otologic literature. Over the next decade, mastoiditis and otosclerosis gradually decreased, while cholesteatoma, noise induced hearing loss, tinnitus and chronic otitis media. Increased From 1978 to 1993, the relative frequency of topics was relatively stable, with the exception of otitis media with effusion and vestibular schwannoma, which increased. From 1993 to now, age related hearing loss and tinnitus have gradually increased, while otitis media with effusion and vestibular schwannoma have diminished.

To validate these observations, statistical analysis was applied. From 1946 to now, labyrinthitis, otosclerosis, chronic otitis media, cholesteatoma, Meniere's disease and mastoiditis

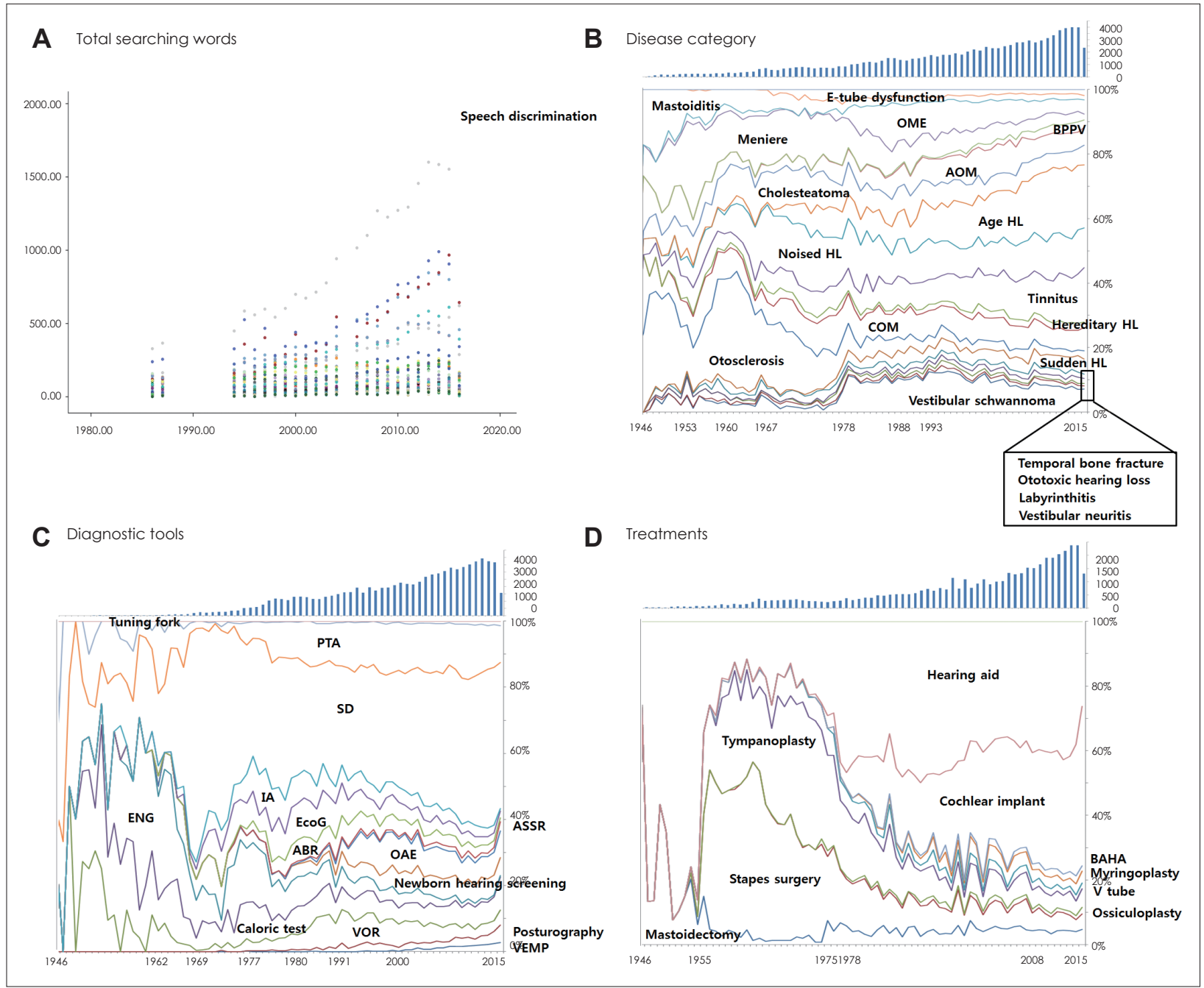

Fig. 1. Changing pattern of published article in otologic field. A: The increasing frequency of each topic in the field of otology. B: The proportion of published articles by disease name. C: The changing proportion of publications based on diagnostic tool from 1946 to now. D: The proportion of published articles based on the treatments provided. 
decreased gradually in the proportion of published articles (rho value $<0$ ). The other topics gradually increased as a result of an expanding number of journals and otology articles. The results for each of the following diagnoses were: ototoxic hearing loss (rho $=0.911)$, age related hearing loss $(\mathrm{rho}=0.972)$ and BPPV (rho=0.949) In each case, the correlation of number of references and time was very high, demonstrating continued increase in total published articles.

\section{The changing pattern of research topics in the 'diagnostic tools' categories}

Fig. 1C shows the changing pattern of published topics in the diagnostic tools' categories. From 1946 to 1960's, pure tone audiometry, speech audiometry, electronystagmography, caloric testing, and vestribulo-ocular reflex were evenly published. In the early 1970's, speech discrimination dramatically increased. At the end of the 1970's, the proportion of pure tone audiometry and electronystagmography increased. However, since then, the proportion of articles on electronystagmography gradually decreased. From 2000 to the present, speech discrimination progressively increased based on word search, so that at present, the proportion of articles which include speech discrimination is equal to almost half of all published articles in this diagnostic tools' category. However, the correlation over time demonstrated the increasing rate was slow (rho=0.409). In contrast, other topics increased rapidly: vestibular evoked myogenic potential ( $r h o=0.828)$, postulography (rho $=0.925)$, otoacoustic emission ( $\mathrm{rho}=0.856$ ), and auditory steady state response (rho $=0.878$ ) even though the absolute frequency of published articles for each was relatively small.

\section{The changing pattern of research topics in the 'treatments' categories}

Fig. 1D shows the changing pattern of published topics in the diagnostic tools' categories. From 1946 to 1955, the most frequently published topic was hearing aid and mastoidectomy in the treatment category. From 1955, to 1978, the number of times tympanoplasty and stapes surgery were mentioned dramatically increased, but they diminished as a proportion of the total publications due to the rapid rise in the number of articles on hearing aid.

After 1978, literature written on cochlear implantation increased tremendously so that the proportions of published articles on tympanoplasty and stapes surgery were decreased as a result. Interestingly hearing aid maintained its proportion of the total published literature, but the rapidly increasing frequency of cochlear implant passed it in frequency in 2015. The correlation of the increased number of cochlear implantation with year was very high $(\mathrm{rho}=0.946)$. And, ossiculoplas- ty, ventilation tube, and bone anchored hearing aid also dramatically increased (rho $=0.836,0.882,0.914)$ even though the total number of articles on these topics was relatively small. Over the same time, the proportion of articles on stapes surgery decreased significantly (rho=-0.246).

\section{Discussion}

In the present study, we examine the changing pattern of published topics, according to disease names, diagnostic tools, and treatment modality. Over the past several decades, there has been a rapid increase in the total number of articles published in the field of otology. In addition, in most of the articles, the authors recommend additional studies to be completed by other investigators or clinicians in the published journals and text books.

Three periods can be identified in the otology literature. The first had to do with evaluation and treatment of infection. Following World War II, the bio-science changed dramatically [8]. The use of penicillin altered the treatment of infectious diseases [9]. In the field of otology, a major pediatric disease, mastoiditis, could now be treated effectively through the prescription of the newly developed antibiotics with good results. As a result, the proportion of articles on topics like mastoiditis, chronic otitis media, and cholesteatoma, represented nearly all of the articles published in this period, form 1945 to 1960 s. Similarly, the proportion of articles on these topics searchable through the related diagnostic tool and treatment modality searches was similarly high in the published journals.

The second time period from the 1960s to the 1990s focused on advances in hearing aids and middle ear surgery. All topics were published almost equally in frequency in this period. The problems associated with most infectious diseases had been resolved using established diagnostic and therapeutic tools. At the end of this period, new innovative tools like cochlear implantation were developed and the results proven [10]. As a result, the main research areas in this third period were focused on cochlear implantation and related topics, including speech discrimination and hearing advances in hearing aids. The spread effect in research is well demonstrated on the continued increase in publications on cochlear implantation, and as a result, the proportion of articles on this topic continues to expand dramatically up to the present. Similarly, over this period, age related hearing loss has gradually increased as a proportion of published articles reflection the increase in the proportion of the population in the older age category.

Its most common use in the past has been to identify the 'most citrated articles' as a means to evaluate relative publishing influences [2-6]. However, this method fails to identify 
the most promising opportunities for future clinical and experimental studies. One reason is that by definition, the most cited articles are retrospective, not current, and therefore don't necessarily reflect the most promising current areas of investigation. Second, the most cited articles don't always reflect the most important trends in research. For example, the most cited study among otolaryngologic articles from 1985 to 2014 was 'facial nerve grading system' by House and Brackmann, published in 1985 [2]. Although an important contribution to otology, it did not indicate an area of additional analysis for others. When the word search methodology of the current approach is applied, facial nerve palsy was relatively insignificant due to very low number of published studies which included this term. Third, there has been no statistical proof that any of the results based on the ranking of citations, the relative ranking of publications, or the change over time effectively predicts future research trends.

To address these limitations in the citation analysis approach, several researchers have applied novel statistical analysis methods to establish prognostic significance.

Sweileh, et al. [11] used Poisson loglinear regression analysis to create a tool which could serve as a reliable predictor. Ranasinghe, et al. [12] used the Cochran-Armitage test for trend analyses. Pearson and Spearman rank correlation analysis was introduced by Eysenbach [13].

We believed a simplified, easy to access method was needed to understand current publications. Fortunately, 'Pubmed. gov' provided the tools needed, allowing searching for "results by year" through the internet on its home page. Downloaded data could then be merged into one data file, and analyzed for significant trends. Although this approach yields huge amounts of information, it too has a major limitation. The results of 'Index Medicus 1946-1965' and 'other journals in multiple languages' weren't included. And, searching by word does not guarantee that the topic was addressed in detail, or served as the focus of the research. In many cases, the word is not central to the investigation itself. For example, recent interesting topics: single sided deafness, hearing preservation, neural plasticity, third window syndrome, migraine, neurovascular cross compression, etc. were major topics today, weren't influence the results of present study. We think dramatically increasing of 'speech discrimination' might be due to common element of recent interesting topics. And, overlap of searching words, multicollinearity, false positive, false negative, etc. were was major limitations to analysis the patterns. For example, 'pure tone audiometry' and 'Bekesy audiometry' were almost identical during study period indicating equivalency, as multicollinearity. If present hypothesis was wrong, over- or underestimate of some searching words were possible. For example, speech discrimination might be over-estimate due to basic word to other searching topics.

Even though these major limitations, we do not believe these reduces the validity of the findings. We hypothesize that this same limitation would apply to all of the words and topics with relatively similar percentages. As a result, the comparative frequency of inclusion would be relatively equivalent for each searched disease, diagnostic tool, or treatment modality. As such, the findings in the review would be directionally correct, particularly over time.

Finally, the results of present study restrictedly suggested changing pattern of past published articles. Prediction of major topics in the future is subscribers' own particular role.

In conclusion, the field of otology has evolved dramatically from prior to World War II to the present. The advances in diagnosis and treatment can be identified through a bibliometric analysis of the changing patterns of published articles using this "suggested new method" of word search. We applied this methodology to analyze the proportion of published articles in the categories of diagnosis, diagnostic tools, and treatment tools in the otologic field. We believe this method and approach can be used by investigators in various departments of Otorhinolaryngology Head and Neck Surgery to identify their research and clinical investigation.

\section{Acknowledgments}

This work was supported by the National Research Foundation of Korea (NRF) grant funded by the Korea government (NRF-2016R1 D1A1B01014128), Republic of Korea.

\section{Conflicts of interest}

The authors have no financial conflicts of interest.

\section{REFERENCES}

1) Swan J. Observations on some points relating to the Physiology and Pathology of the Ear. Med Chir Trans 1818;9(Pt 2):422-6.

2) Lenzi R, Fortunato S, Muscatello L. Top-cited articles of the last 30 years (1985-2014) in otolaryngology-head and neck surgery. J Laryngol Otol 2016;130:121-7.

3) Fenton JE, Roy D, Hughes JP, Jones AS. A century of citation classics in otolaryngology-head and neck surgery journals. J Laryngol Otol 2002;116:494-8.

4) Jafarzadeh H, Sarraf Shirazi A, Andersson L. The most-cited articles in dental, oral, and maxillofacial traumatology during 64 years. Dent Traumatol 2015;31:350-60.

5) Coelho DH, Edelmayer LW, Fenton JE. A century of citation classics in otolaryngology-head and neck surgery journals revisited. Laryngoscope 2014;124:1358-62.

6) Ruben RJ. The most cited and requested articles published in the International Journal of Pediatric Otorhinolaryngology. Int J Pediatr Otorhinolaryngol 2009;73:513-22.

7) De S, Jones T, Brazier H, Jones AS, Fenton JE. The accuracy of MEDLINE and Journal contents pages for papers published in Clinical Otolaryngology. Clin Otolaryngol Allied Sci 2001;26:39-42.

8) Bud R. Innovators, deep fermentation and antibiotics: promoting applied science before and after the Second World War. Dynamis 2011; 
31:323-41.

9) Yambao CV, Bongco F. Penicillin in acute mastoiditis with report of three cases recovered. J Philipp Med Assoc 1948;24:119-22.

10) Albernaz PL. History of cochlear implants. Braz J Otorhinolaryngol 2015;81:124-5.

11) Sweileh WM, Zyoud SH, Al-Jabi SW, Sawalha AF, Shraim NY. Drinking and recreational water-related diseases: a bibliometric analysis (1980-2015). Ann Occup Environ Med 2016;28:40.
12) Ranasinghe I, Shojaee A, Bikdeli B, Gupta A, Chen R, Ross JS, et al. Poorly cited articles in peer-reviewed cardiovascular journals from 1997 to 2007: analysis of 5-year citation rates. Circulation 2015;131: 1755-62.

13) Eysenbach G. Can tweets predict citations? Metrics of social impact based on Twitter and correlation with traditional metrics of scientific impact. J Med Internet Res 2011;13:e123. 